\title{
Mehr als nur ein weiterer Ratgeber
}

\section{Gregor Berger}

PD Dr. med., UniversitätsSpital Zürich

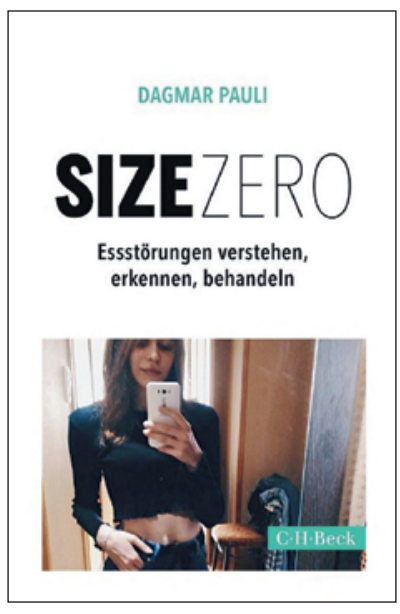

Dagmar Pauli

SIZE ZERO - Essstörungen verstehen, erkennen, behandeln

München: C.H. Beck; 2018.

223 Seiten. $26.90 \mathrm{CHF}$.

ISBN 978-3406726675

\section{Verzerrte Selbstbilder auf dem Boden einer «essgestörten Gesellschaft»}

Das neu erschienene Buch «SIZE ZERO - Essstörungen verstehen, erkennen und behandeln» der international renommierten Essstörungsspezialistin Dagmar Pauli ist mehr als ein weiterer Ratgeber zum Thema Essstörungen. Das Buch setzt sich kritisch aus gesellschaftlicher Perspektive mit dem verzerrten Selbstbild einer heranwachsenden Jugend auseinander und erläutert gleichzeitig prägnant dessen Folgen für das Individuum. Die Autorin erörtert den Zusam-

\section{Das Buch setzt sich kritisch mit dem verzerrten Selbstbild einer heranwachsenden Jugend auseinander.}

menhang einer «essgestörten Gesellschaft» mit den Krankheitsbildern der verschiedenen klinischen und subklinischen Essstörungen. Ihr ist es in einzigartiger Weise gelungen, diesen komplexen Sachverhalt in einer einfachen, gut nachvollziehbaren Sprache und mit ausgesprochener Sachlichkeit aufzuarbeiten, bei gleichzeitig hoher Sensibilität für die Betroffenen und deren Familien. Angesprochen sind auch Fachpersonen und Menschen, die direkt oder indirekt mit gefährdeten Kindern und Jugendlichen zusammenarbeiten.

\section{Gesellschaftliche Problematik}

Im ersten Teil des Buches zeigt die Autorin mit hoher Deutlichkeit auf, dass grosse Teile unserer Gesellschaft an den Symptomen einer Essstörung leiden. Eine ungesunde Ess- und Körperkultur wird tagtäglich in verschiedensten Medien propagiert und angepriesen, wodurch die Heranwachsenden mit «kranken Idealen» konfrontiert werden. Diese animieren

\section{Grosse Teile unserer Gesellschaft leiden an den Symptomen einer Essstörung.}

sie zu ungesunden Essgewohnheiten oder Diäten, die wiederum zu Essstörungen und Adipositas beitragen. Pauli kritisiert zu Recht, dass wir als Gesellschaft nicht genug unternehmen, die Medien- und Modewelt in einem verbindlichen Dialog zu verpflichten, um den Kindern und Jugendlichen die Chance zu geben, eine gesunde Beziehung zu ihrem Körper und dem Essen aufzubauen. Sie fordert hier ein Handeln auf verschiedenen Ebenen: von präventiven Massnahmen in der Schule, der Identifizierung von Risikopopulationen bis hin zu gesetzlichen Rahmenbedingungen, die der "gesellschaftlichen Essstörung» Grenzen setzen.

\section{Aufräumen mit Mythen}

Im zweiten Teil ihres Buches erklärt die Autorin die komplexen und ineinander verwobenen Ursachen, die Essstörungen auslösen und aufrechterhalten können. Sie räumt mit Mythen über Essstörungen auf, wobei sie insbesondere die Familie aus dem Schussfeld der Schuldzuweisung nimmt und die Essstörung als gemeinsamen «Feind» in den Mittelpunkt aller therapeutischen Bemühungen stellt. So zeigt sie einen Weg auf, diese hartnäckige Erkrankung gemeinsam anzugehen, zu überwinden und 
eine Chronifizierung zu vermeiden. Sie ist eine Advokatin der Früherkennung und Frühintervention und zeigt konkrete Schritte auf, wie eine solche verbessert werden kann. Schliesslich gelingt es Pauli im dritten

\section{Die Autorin nimmt insbesondere die Familie aus dem Schussfeld der Schuldzuweisung.}

Teil des Buches, die gegenwärtigen Erkenntnisse zur Behandlungen der Essstörungen in einer für mich bisher nicht dagewesenen verständlichen Art zusammenzufassen. Besonders gefallen hat mir das Kapitel 13, wo sie in einem Brief an die Betroffenen formuliert, was sie von ihren vielen hundert Patientinnen und Patienten gelernt hat, deren Ängste und Sorgen sie mit grosser Sensibilität versteht und einfühlsam, aber auch klar und direkt formuliert aufnimmt. Darauf folgt ein Kapitel für die Eltern und die Familie, die sich häufig in einem Teufelskreis befinden, aus dem das ganze System kaum ohne Hilfe herauskommt. Hier zeigt die Autorin Wege und Grundhaltungen auf, die allen Beteiligten helfen können, diesen oft steinigen Weg aus der Krankheit proaktiv und erfolgreich zu bewältigen. Pauli vermeidet es, ein «Betty Bossi»-Rezept zur Behandlung der Essstörungen zu geben, sondern vermittelt vielmehr eine Grundhaltung im Umgang mit dieser komplexen Störung.

\section{Appell an die Medien- und Modewelt}

Das Buch ist eine lohnenswerte Lektüre für Betroffene, deren Familie, aber auch für Fachleute und Interessierte, die mit Kindern und Jugendlichen arbeiten. Es ist ebenso ein Appell an die Medienwelt und Modebranche, endlich ihre Verantwortung wahrzunehmen und ein gesundes Körperbild für Heranwachsende zu bewerben, das die Vielfalt der Jugendlichen und jungen Erwachsenen repräsentiert, anstatt nur ein künstlich «retouchiertes», überzeichnetes, unerreichbares, krankes und verzerrtes «Ideal» des erfolgreichen androgynen Jugendlichen darzustellen. Gesundheitspolitiker, Medien-

Ein äusserst lesenswertes Buch, das zur Entstigmatisierung und womöglich lebensrettenden frühzeitigen Behandlung beiträgt.

schaffende und Funktionäre von Sportverbänden, insbesondere von Risikosportarten, wie Kunstturnen, Eiskunstlaufen oder Ballett, nimmt sie in die Pflicht, die Kinder und Jugendlichen vor krankmachenden Exzessen zu schützen. Es handelt sich um ein äusserst lesenswertes Buch, das zur Entstigmatisierung und unter Umständen lebensrettenden frühzeitigen Behandlung dieser schwerwiegenden Krankheit beiträgt, die immer noch eine der höchsten Sterberaten von allen psychischen Störungen überhaupt aufweist. 